\title{
Risk factors for mortality from imported falciparum malaria in the United Kingdom over 20 years: an observational study
}

In this Research article by Anna M Checkley and colleagues (BMJ 2012;344:e2116, doi:10.1136/bmj.e2116), the author for correspondence should have been A M Checkley 